\title{
Revisión
}

\section{Cáncer de próstata: práctica clínica basada en la evidencia}

\author{
Leonardo Oliveira Reis, André Deeke Sasse, Wagner Eduardo Matheus, Fernandes Denardi, \\ Rafael Mamprim Stopiglia, Maurício Moreira da Silva, Ubirajara Ferreira
}

\author{
Sección de Urología Oncológica. Discipline of Urology, Universidad de Campinas - Unicamp, Campinas - SP - \\ Brazil
}

\begin{abstract}
Resumen
Objetivo: La medicina basada en la evidencia permite la mejor evidencia clínica externa de la que se dispone respecto a las búsquedas sistemáticas en la literatura para determinar la amplitud de su recomendación. Esta guía clínica tiene la intención de asistir a médicos y profesionales de la salud en decisiones clínicas en relación con el tratamiento del cáncer de próstata, particularmente en urología, oncología clínica y radioterapia.

Métodos: Las publicaciones usadas como fuentes de información fueron obtenidas de búsquedas en bases electrónicas como CENTRAL (Cochrane Central Register of Controlled Clinical Trials) y MEDLINE (online). Cada punto de esta guía clínica deriva de una pregunta original distribuida entre los participantes. Las estrategias de búsqueda fueron preparadas para elegir estudios presentando la mejor calidad metodológica, de acuerdo a los niveles de evidencia predefinidos.

Resultados: Todas las recomendaciones fueron seguidas por un nivel de evidencia (NE) y un grado de recomendación (GR). Hemos usado un sistema de ranking formal para ayudar al lector a juzgar la amplitud de la evidencia.

Conclusiones: Los parámetros existentes deberían ser vistos como guías clínicas de conducta. El ensayo final en el cual el proceso clínico o plan de tratamiento es más conveniente para un paciente debe ser hecho por un médico, quien discutiría las opciones de tratamiento disponibles con el paciente de acuerdo con el diagnóstico
\end{abstract}

Palabras clave: Guía Clínica. Medicina basada en la evidencia, Cáncer de próstata.

\section{Prostate cancer: evidence based clinical practice}

\section{Abstract}

Objectives: Evidence-based medicine allows the best available external clinical evidence from systematic literature research to be graded in order to determine the strength of its recommendation. This guideline aims to assist physicians and health professionals in clinical decisions related to prostate cancer treatment, particularly in urology, clinical oncology and radiotherapy.

Methods: The publications used as information sources were obtained from structured data search in electronic databases, such as CENTRAL (Cochrane Central Register of Controlled Clinical Trials) and MEDLINE (online). Each item of this guideline derived from an original question which was distributed to the participants. Search strategies were prepared to select the studies presenting the best methodological quality, according to predefined levels of evidence.

Results: All the recommendations were followed by a level of evidence (LE) and a degree of recommendation (DR). We used a formal ranking system to help the reader to judge the strength of the evidence behind the results published in support of each recommendation. Conclusions: The existing parameters should be viewed as guidelines of conduct. The final trial on which the clinical procedure or treatment plan is most suitable for a particular patient should be done by a physician, who should discuss the available treatment options with the patient according to the diagnosis.

Keywords: Guideline. Evidence-based medicine. Prostate cancer.

$\mathrm{L}^{2}$ a práctica de la medicina basada en la evidencia requiere a los clínicos integrar la experiencia clínica individual con la mejor evidencia clínica externa disponible frente a las búsquedas sistemáticas ${ }^{1}$. Elegir la mejor evidencia externa clínica disponible confía el asesoramiento del diseño del estudio y calidad del papel para determinar su fuerza ${ }^{2}$. Hay una jerarquía general de evidencia con ensayos clínicos controlados y meta análisis a la cabeza y opinión de expertos en la base. Un sistema comúnmente usado es el Oxford Centre for Evidence-based Medicine Levels of Evidence ${ }^{3}$, que permite a la literatura gradar en aras de determinar la fuerza de su recomendación. A veces un ensayo clínico controlado no está disponible para un tema de interés y la mejor evidencia disponible podría venir en la forma de otro estudio. Esta guía clínica se propone asistir a médi$\cos$ y profesionales de la salud en las decisiones 
relacionadas con el tratamiento del cáncer de próstata, particularmente en urología, oncología médica y oncología radioterápica. Las publicaciones usadas como fuentes de información fueron obtenidas en búsquedas estructuradas de bases de datos electrónicas como CENTRAL (Cochrane Central Register of Controlled Clinical Trials) y MEDLINE (online). Cada punto de esta guía clínica deriva de una pregunta original distribuida entre los participantes. Las estrategias de búsqueda fueron preparadas para elegir estudios presentando la mejor calidad metodológica, de acuerdo a los niveles de evidencia predefinidos. Versiones preliminares de estas recomendaciones fueron presentadas a especialistas en áreas envueltos en el desarrollo universitario (urología, oncología médica y oncología radioterapia) y tras ello se mantuvieron encuentros para la evaluación final, incorporación de sugerencias y aprobarlas por escrito. Todas las recomendaciones fueron seguidas por un nivel de evidencia (NE) y por un grado de recomendación (GR). Hemos usado un sistema de ranking formal para ayudar al lector a juzgar la amplitud de la evidencia. Se desarrollaron muchos tipos de gradación de escalas y se usaron sistemas simplificados de acuerdo al tipo de protocolo (tratamiento o diagnóstico), haciéndolo fácil de leer, como en la Tabla 1. Los parámetros existentes. La adherencia a todos estos parámetros no puede asegurar un resultado clínico satisfactorio en todos los casos. El ensayo final en el cual el procedimiento clínico o plan de tratamiento es más adecuado

Tabla 1. Escala de graduación de niveles de evidencia y grado de recomendación

\begin{tabular}{llll}
\hline $\begin{array}{l}\text { Nivel de } \\
\text { evidencia }\end{array}$ & $\begin{array}{l}\text { Grado de } \\
\text { recomendación }\end{array}$ & Tratamiento & Diagnóstico \\
\hline 1 & $\begin{array}{l}\text { A (fuerte) } \\
\text { Sistemática } \\
\text { Metaanálisis } \\
\text { Randomizado } \\
\text { Metaanálisis }\end{array}$ & $\begin{array}{l}\text { Revisión } \\
\text { Sistemática } \\
\text { Corte validado }\end{array}$ & Revisión \\
& A (fuerte) & Randomizado \\
& & (pequena muestra) & Corte \\
3 & B (moderado) & Prospectivo & Selección No \\
& & & conservadora \\
4 & C (débil) & Retrospectivo & Casos y Controles \\
5 & D (muy débil) & Serie de Casos & \\
& Opinión de & Series de casos & \\
& Expertos & Opinión de Expertos & \\
& Preclínico & & \\
& & &
\end{tabular}

para cada paciente debería ser hecho por un médico quien discute en este caso las diferentes opciones terapéuticas con el paciente, de acuerdo con el diagnóstico. Sin embargo, sería prudente que las diferencias significativas en la práctica en relación a estas recomendaciones sean justificadas y sus razones documentadas con propiedad. Esta guía clínica fue producida en 2009 y está sujeta a revisión tan pronto como se disponga de una nueva evidencia. Se ha programado una puesta al día para Noviembre de 2009.

\section{CLASIFICACIÓN DE RIESGOS}

La selección de pacientes para tratamientos específicos se hace usualmente mediante la subdivisión de riesgo de recurrencia, que se describe a continuación ${ }^{4}$ :

- Riesgo Bajo: T1-T2a, Gleason 2 a 6, PSA menor de 10.

- Riesgo Intermedio: T2b-T3a, Gleason 7, PSA 10 a 20.

- Riesgo Alto: Mayor que T3b, Gleason mayor que 7, PSA mayor de 20.

Una revisión sistemática de la Cochrane Collaboration actualizada en Mayo de 2006 incluía resultados de dos grandes estudios randomizados ${ }^{5}$ con 55512 participantes que fueron remitidos tras tacto rectal (TR) y/o PSA y/o ecografía transrectal.

La existencia de serios problemas metodológicos en el diseño de los estudios incluía evitar conclusiones definitivas, pero el meta análisis mostró diferencias no significativas en la mortalidad entre la población seleccionada y la no seleccionada (RR 1,01, 95\% CI 0,8-1,29).

Hay otros dos grandes estudios randomizados en progreso sobre el tema sin resultados disponibles de momento. Los resultados de estos estudios deberían ser presentados en los próximos años y deberían determinar como el screening debería ser ofrecido al público. Hoy no hay evidencia que el screening con TR y/o PSA y/o Ecografía Próstata sea efectivo en la reducción de la mortalidad asociada al cáncer de próstata y que aumente su supervivencia. 
Hasta la publicación de futuros estudios, recomendamos la realización de un PSA anual y un Tacto Rectal para la búsqueda de un cáncer de próstata en varones entre 50 y 80 años. En varones con familiares de primer grado afectos lo recomendamos a partir de los 45 años de edad (NE 5, GR D).

\section{DIAGNÓSTICO}

La biopsia para el diagnóstico de cáncer de próstata debe practicarse en pacientes con las siguientes características:

1. Tacto Rectas sospechoso ${ }^{6}$ (NE 1, GR A).

2. PSA mayor de $2.5 \mathrm{ng} / \mathrm{ml}$ en pacientes hasta 55 años $^{7}$ (NE 2, GR A).

3. PSA mayor de $4 \mathrm{ng} / \mathrm{ml}$ en pacientes mayores de 55 años $^{8}$ (NE 2, GR A).

4. Velocidad de PSA mayor de $0,75 \mathrm{ng} / \mathrm{ml} / \mathrm{añno}^{9}$ (NE 3, GR B).

5. Densidad de PSA mayor de $0,15 \mathrm{ng} / \mathrm{ml}^{10}(\mathrm{NE}$ 3, GR B).

6. Relación de PSA total y PSA libre menor de $18 \%$ cuando el PSA es mayor de $2 \mathrm{ng} / \mathrm{ml}^{11}$ ( $\mathrm{NE}$ 1, GR A).

La biopsia debe ser realizada con la adecuada profilaxis antibiótica. Los antibióticos deben ser económicos, de amplio espectro y dirigidos al tejido prostático y con escasos efectos adversos ${ }^{12}$ (NE 2, GR A). Recomendamos el uso de fluorquinolonas durante un día.

La biopsia debe ser guiada por ultrasonidos (US) con más de 10 fragmentos incluyendo las caras latero laterales ${ }^{13}$ (NE 2, GR A) con anestesia local peri prostática (de fácil manejo, efectiva y baja morbilidad) ${ }^{14}$ (NE 2, GR A)

La re biopsia debería ser realizada con un intervalo mínimo de 6 a 8 semanas en pacientes con las siguientes características:

1. Persistencia de PSA elevado o creciendo a una velocidad superior a $0.75 \mathrm{ng} / \mathrm{ml}^{15}$ (NE 3, GR B).

2. Con sospecha pero sin enfermedad patológica confirmada (proliferación acinar atípica). La biopsia debe ser sistemática y sobre el área afectada $^{16}$ (NE 3, GR B).

Excepto en situaciones especiales, el límite máximo recomendado es de 4 biopsias, usando la técnica apropiada. La biopsia de saturación incrementa la probabilidad de positividad y provee datos de extensión extracapsular ${ }^{17}$ (NE 2, GR A).

El uso del Doppler incrementa la efectividad del ultrasonido rectal para identificar cáncer y debería usarse cuando sea posible, especialmente durante la rebiopsia ${ }^{18}$ (NE 3, GR B). El contraste por ultrasonido puede incrementar la sensibilidad Doppler ${ }^{19}$ (NE 3, GR B).

Espectroscopia junto a la Resonancia Magnética Endorectal puede dirigir en áreas sospechosas en pacientes con elevación de PSA y biopsias previas negativas $^{20}$ (NE 3, GR B).

\section{INVESTIGACIONES}

Las investigaciones hechas para priorizar el tratamiento están en relación con la detección de metástasis a distancia y plan de tratamiento.

Pruebas de laboratorio:

1. Fosfatasa Alcalina (FA) y su fracción ósea total: Tiene una sensibilidad del 65\%; la fracción ósea tiene una mayor especificidad diagnóstica cuando se compara con el total de Fosfatasa Alcalina (90 vs. 57\%). Con un punto de corte de fracción ósea total de $30 \mathrm{ng} / \mathrm{ml}$, las metástasis óseas se detectan en un $88 \%$ con una especificidad del $100 \%$. Recomendamos la fracción ósea total en los pacientes con una baja probabilidad de presentar metástasis óseas (riesgo bajo e intermedio) y sin síntomas de cáncer óseo (T1-2 y Gleason menor o igual a 7 y PSA inferior a $20 \mathrm{ng} / \mathrm{ml})^{21}$ (NE 2, GR A).

2. Otros: osteocalcinina, procolágeno I, excreción de calcio urinario, hidroxiprolina, sialoproteína ósea, osteopontina plasmática entre otros, de los que no se ha probado su eficacia y no se recomiendan para el diagnóstico de metástasis ósea en paciente con un nuevo diagnóstico de cáncer de próstata.

Imagen:

No hay evidencia que indique ningún tipo de ultrasonido en el estadiaje de cáncer de próstata. Pacientes con niveles bajos de PSA y sin dolor óseo no tienen indicación de gammagrafía ósea ${ }^{22}$. La recomendamos con pacientes con PSA superior a 20 $\mathrm{ng} / \mathrm{ml}$ (con independencia del estadio $\mathrm{T}$ y del grado Gleason) y/o enfermedad local avanzada (T3 o T4) y/o Gleason superior a 7 y/o dolor óseo y/o elevación de los niveles de $\mathrm{FA}^{23}$ (NE 2, GR A). La Resonancia Magnética Endorectal (RMN) con bovina promete una mejor resolución espacial que cualquier método disponible en la actualidad. Tres técnicas mayores han sido estudiadas en pacientes con cáncer de próstata, pero no hay evidencia que justifique su aplicación de rutina, a saber: RMN T2, RMN y Espectroscopia dinámica por $\mathrm{RMN}^{24}$. Concluimos 
que la RMN con bovina endorectal puede ser usada en los siguientes pacientes: T1-T2 y Gleason inferior a 7 y PSA superior a $2 \mathrm{ng} / \mathrm{ml}$ o T1-T2, Gleason 8-10 y PSA inferior a $20 \mathrm{ng} / \mathrm{ml}^{25}$. Dicha técnica puede, asimismo, ayudar en la planificación quirúrgica de pacientes T1$\mathrm{T} 2$, Gleason superior a 7, PSA superior a $20 \mathrm{ng} / \mathrm{ml}$ o con invasión de vesículas seminales o vejiga.

Una tomografía computada tiene menor precisión que la disección ganglionar del abdomen y pelvis, tiene valor limitado en el estadiaje local y la disección nodal debe reservarse a casos de pacientes de alto riesgo ${ }^{26}$. El papel de la Tomografía de Emisión de Positrones (PET) ha sido evaluado en muchos estudios en el estadiaje de cáncer de próstata. El PET-FDR no está todavía en uso $^{27}$ y otros radioisótopos están bajo investigación.

\section{TRATAMIENTO}

\section{Vigilancia activa}

Pacientes con cáncer de próstata de bajo riesgo (Gleason menor de 7, PSA menor de $10 \mathrm{ng} / \mathrm{ml}$, TlcT2a) y enfermedad con volumen inferior a $0.5 \mathrm{~cm} 3$ evaluados mediante biopsia, deben ser seguidos mediante PSA trimestral y biopsia anual. La enfermedad de mínimos volumen se define por: Densidad de PSA menor de 0.10 , menos de 2 fragmentos positivos en toda la muestra o menos de 50\% de los fragmentos afectos o Gleason inferior a $7^{28}$ (NE 3, GR B)

La elevación de PSA indica la necesidad de re biopsia. El incremento en el PSA total de más de 2 $\mathrm{mg} / \mathrm{ml} / \mathrm{año}$, Tiempo de doblaje de PSA menor de 3 meses o empeoramiento del Gleason significa fallo de la vigilancia activa ${ }^{29,30}$ (NE 3, GR B). Deberíamos de esta manera seleccionar pacientes para seguimiento con los siguientes criterios:

- Mas de 65 años

- Probabilidad de volumen tumoral hasta un máximo de $0,5 \mathrm{~cm}^{3}$

- Ausencia de Gleason de 4 o 5

- Densidad de PSA menor de 0,1

- Máximo del 50\% de los fragmentos afectados

\section{Cirugia}

La cirugía radical está indicada en tumores hasta T2c con esperanza de vida mayor de 10 años y raramente se ofrece a hombres mayores de 70 $a_{n}{ }^{31}$. La cirugía puede también estar indicada en paciente jóvenes con tumores T3 ${ }^{32}$ (NE 5, GR D). No hay una buena evidencia científica que demuestre la superioridad de una técnica respecto a otra en cuanto al control de la enfermedad. El acceso retropúbico preconizado por Walsh es la más ampliamente usada y entre sus ventajas está la posibilidad de la linfadenectomía simultánea y la exención de instrumentos especiales

La linfadenectomía clásica consiste en quitar el tejido linfoide de la vena iliaca externa en ambos lados de la cavidad pélvica, pared medial de la vejiga, suelo pélvico, ligamento de Cooper y arteria hipogástrica. Otras disecciones están en discusión por el riesgo de linfedema y solo se ejecutan en caso de alto riesgo de metástasis linfoide.

Pacientes con PSA menor de $10 \mathrm{ng} / \mathrm{ml}$ o Gleason inferior a 7 no necesitan linfadenectomía de forma rutinaria $^{34}$ (NE 5, GR D).

La vida media del PSA es de 2.6 días, indetectable desde dos a cuatro semanas después de la cirugía.

\section{Radioterapia}

Bajo riesgo

La radioterapia conformada con plan computado (3D CRT) es una opción en pacientes con cáncer de próstata de bajo riesgo. La dosis de 74-78 Gy (2 Gy por día) en un acelerador de alta energía fue superior en términos de control de la enfermedad comparada con dosis más bajas (64 o 70 Gy) ${ }^{35}$ (NE 2, GR A).

Otra opción, con similar control de la enfermedad es la braquiterapia de altas dosis (HRB) en monodosis, una fuente de Iridio 192 con dosis de 38 Gy (4 fracciones de 9.5 Gy en dos dias) o implante permanente $^{36}$ (NE 3, GR B).

\section{Intermedio $y$ alto riesgo}

Hay una opción de tratamiento para paciente con riesgo de alto e intermedio de cáncer de próstata y vesículas seminales divididas en dos fases, con un máximo de 50.4 Gy solo en la próstata, con una dosis final de 74-78 Gy (2 Gy por día) en un acelerador lineal de alta energía ${ }^{35}$ (NE 2, GR A)

Puede ofrecerse una dosis alta de braquiterapia como radioterapia externa con una fuente de Iridio 192 con dosis de 19 Gy (2 fracciones de 9.5 en un dic) ${ }^{37}$ (NE 3, GR B).

Radioterapia adyuvante postprostatectomía radical

En pacientes con márgenes positivos, enfermedad extracapsular o invasión de vesículas seminales, la radioterapia post operatoria has demostrado mejoría en el tiempo libre de supervivencia en un estudio prospectivo randomizado ${ }^{38}$. 
La radioterapia externa puede ser indicada en estadios simples con una dosis final de 66 Gy (2 Gy por día) en el acelerador lineal de alta energía ${ }^{38}$ (NE 1, GR A).

\section{Hormonoterapia}

La hormonoterapia adyuvante o neo adyuvante previo a la prostatectomía fue evaluado usando una revisión sistemática con meta análisis de 13 ensa$\operatorname{yos}^{39}$. No hay diferencias en cuanto a la supervivencia global y supervivencia enfermedad específica, sin embargo un estudio mostró mejores tasas estadísticas de supervivencia libre de enfermedad y de enfermedad específica con la adición de tratamiento adyuvante. La misma revisión sistemática con meta análisis de 4 estudios evaluando ${ }^{39}$ terapias hormonales neo adyuvantes combinadas radioterapia mostraron mejorías significativas in supervivencia libre de enfermedad pero no en cuanto a supervivencia global.

Un tratamiento hormonal antes de la radiación incrementa el control locorregional de pacientes de riesgo intermedio y alto. Seis meses de tratamiento neo adyuvante parece ser el más efectivo en reducir la recurrencia de cáncer de próstata frente a 3 meses de tratamiento ${ }^{40}$.

Adicionalmente, la misma revisión sistemática de terapia adyuvante hormonal fue usada previamente a la radioterapia en 4 estudios pacientes de moderado a alto riesgo ${ }^{39}$.

Un meta análisis de resultados mostró mejorías en supervivencia general a los 5 y 10 años, supervivencia enfermedad específica y supervivencia libre de enfermedad. Un estudio prospectivo randomizado de 970 pacientes de intermedio y alto riesgo ${ }^{41}$ para recibir tratamiento de seis meses o 3 años de tratamiento adyuvante con LHRH. Se observó que los tratamientos de 6 meses presentaron peor supervivencia global y tasas más altas de progresión.

No recomendamos tratamiento adyuvante o neoadyuvante antes de la prostatectomía radical por la falta de evidencia de beneficio clínico (NE1, GR A). Recomendamos hormonoterapia adyuvante combinada con radioterapia en pacientes de moderado y alto riesgo con enfermedad localizada por un periodo de 3 años (NE 1, GR A).

\section{Tratamiento del fallo bioquímico}

Después de la Radioterapia, el fallo bioquímico se define por la RTOG-ASTRO (Conferencia de Phoenix 2006) como una elevación de PSA de 2 ng/ml o más sobre el PSA nadir después de la RT con o sin tratamiento hormonal concomitante o tres incrementos consecutivos de PSA en un intervalo mínimo de tres meses ${ }^{42}$ (NE 5, GR D).

También se considera fallo bioquímico cuando los pacientes muestran elevación de PSA después de la cirugía (sobre $0.2 \mathrm{ng} / \mathrm{ml})^{43}$. Radioterapia de rescate debería ofrecerse, como se ha descrito anteriormente, como RT adyuvante después de la prostatectomía (NE 4, GR C)

No hay evidencia que el tratamiento del fallo bioquímico cambie el pronóstico del paciente. A pesar de ello, la introducción de la terapia hormonal, terapia de rescate local (cirugía o radioterapia) u observación clínica debe ser ofrecidos a los pacientes.

\section{Tratamiento de la enfermedad metastásica}

\section{Hormonoterapia}

Resultados de una revisión sistemática muestran que el bloqueo androgénico completo no añade beneficios clínicos significativos en relación con el bloqueo simple (beneficio absoluto del $2 \%$ en 5 años, con un intervalo de confianza del 0 al 5\%) ${ }^{44}$.

Otra revisión sistemática de la literatura con análisis de 24 estudios randomizados muestra que le supervivencia global es similar entre los pacientes tratados mediante orquiectomía o con análogos de $\mathrm{LHRH}^{45}$.

El uso de bloqueo androgénico intermitente ha demostrado ser tan efectivo como el continuo en pacientes con enfermedad metastásica en estudios randomizados ${ }^{46}$

Por ello, recomendamos las siguientes dosis como tratamiento inicial de bloqueo androgénico (NE1, GR A): goserelina 3,6 mg / leuprolide 7,5 mg cada 28 días o goserelina 10,8 mg / leuprolide 22,5 mg cada tres meses.

Si después de 6 meses de tratamiento con bloqueo androgénico, el PSA desciende a menos de $4 \mathrm{ng} / \mathrm{ml}$ el uso de análogos de LHRH debe ser suspendido hasta que el futuro nivel de PSA aumente a más de 10 $\mathrm{ng} / \mathrm{ml}$, momento en el que el análogo debe ser reintroducido $^{46}$ (NE 2, GR A).

Estudios prospectivos no randomizados muestran que en pacientes con enfermedad progresiva después del bloqueo androgénico permiten un descenso de PSA y un control temporal de la enfermedad con aceptable control de toxicidad ${ }^{47}$. No hay estudios que demuestren aumento de supervivencia usando esta estrategia. 
En pacientes con enfermedad progresiva después del bloqueo androgénico, recomendamos su mantenimiento con estrógenos o combinación periférica de antiandrógenos ( $\mathrm{NE} \mathrm{3,} \mathrm{GR} \mathrm{B)} \mathrm{con} \mathrm{las}$ siguientes dosis: flutamida $250 \mathrm{mg}$ cada 8 horas o bicalutamida $50 \mathrm{mg}$ una vez al día, o ciproterona $100 \mathrm{mg}$ al día o dietilestilbestrol $1 \mathrm{mg}$ al día.

\section{Guimioterapia}

En pacientes con enfermedad metastásica refractaria al tratamiento, un estudio prospectivo randomizado mostró una pequeña ganancia de vida con el uso de docetaxel en comparación con mitoxantrón ${ }^{48}$ asociado con prednisona.

Todos los pacientes permanecieron con el bloqueo androgénico durante el tratamiento con quimioterapia. Es por ello que, recomendamos quimioterapia en pacientes con cáncer metastático refractario al tratamiento hormonal con docetaxel (75 $\mathrm{mg} / \mathrm{m}^{2}$ cada 21 días) combinado con prednisona 5 mg dos veces al día ${ }^{48}$ (NE 2, GR A).

Los pacientes deberían continuar bloqueo androgénico e inicialmente presentar un índice de Karnofsky de al menos 60\%. Antes de cada sesión de quimioterapia, los pacientes deben ser evaluados por el médico responsable con una monitorización de recuentos celulares en sangre, creatinina y enzimas hepáticas.

\section{Otras terapias paliativas}

La resección transuretral de próstata debe ser indicada como tratamiento paliativo en pacientes con síntomas urinarios obstructivos.

En pacientes con dolor óseo localizado, la radioterapia debe ser administrada a dosis de 30 Gy (en diez fracciones) en el área dolorosa con intención paliativa $^{49}$ (NE 2, GR A).

Referente a los pacientes refractarios al tratamiento hormonal con metástasis óseas dolorosas, un estudio prospectivo randomizado mostró menor incidencia de efectos adversos con el uso de ácido zoledrónico ${ }^{50}$. No hay estudios que muestren beneficios clínicos respecto a otros bifosfonatos. Por consiguiente, recomendamos el uso de ácido zoledrónico $4 \mathrm{mg}$ cada 28 días en pacientes refractarios al tratamiento hormonal y metástasis óseas ${ }^{50}$ (NE 2, GR A).

Los pacientes deben ser evaluados antes de cada dosis con monitorización mensual de creatinina y calcio sérico. Los pacientes deben recibir diariamente suplementos de calcio y vitamina D.
En pacientes con dolor óseo refractario al citado anteriormente, el uso de samario-153 debe considerarse. Un estudio prospectivo randomizado mostró mejoría en el dolor y calidad de vida en pacientes refractarios al tratamiento hormonal ${ }^{51}$ (NE 2, GR A).

\section{SEGUIMIENTO}

Después de la Prostatectomía Radical o Radioterapia, el examen físico y el nivel de PSA cada 3 meses durante el primer año, para pasar a ser bianual a partir del segundo año (NE 5, GR D).

El bloqueo hormonal completo debe ser monitorizado con densitometría ósea cada 12 meses para valorar la osteoporosis durante el periodo de bloqueo $^{52}$ (NE 5, GR D).

\section{REFERENCIAS}

1. Sackett DL, Rosenbe DR, WM, Gray JA, Haynes RB, Richardson WS Evidence based medicine: What it is and what it isn't. BMJ 1996, 312(7023): 71-72.

2. Petrisor BA, Keating J, Schemitsch E. Grading the evidence: Levels of evidence and grades of recommendation. Injury 2006, 37(4):321-327.

3. Oxford Centre for Evidence-based Medicine Levels of Evidence. In: Centre for Evidence-Based Medicine, 2001. www.cemb.net/ index.aspx?o=1025.

4. D’Amico AV, Whittington R, Malkowicz SB, Schultz D, Blank K, Broderick GA, et al. Biochemical outcome after radical prostatectomy, external beam radiation therapy, or interstitial radiation therapy for clinically localized prostate cancer. Jama. 1998 Sep 16;280(11):969-974

5. Ilic D, O'Connor D, Green S, Wilt T. Screening for prostate cancer. Cochrane Database Syst Rev. 2006;3:CD004720.

6. Issa MM, Zasada W, Ward K, Hall JA, Petros JA, Ritenour CW, et al. The value of digital rectal examination as a predictor of prostate cancer diagnosis among United States Veterans referred for prostate biopsy. Cancer detection and prevention. 2006;30(3):269-275.

7. Ashley T. Using predictive value, sensitivity and specificity to interpret laboratory tests: PSA for the diagnosis of prostate cancer. Journal of insurance medicine (New York, NY. 2005;37 (4):261-263.

8. Maattanen L, Hakama M, Tammela TL, Ruutu M, Ala-Opas M, Juusela H, et al. Specificity of serum prostate-specific antigen determination in the Finnish prostate cancer screening trial. British journal of cancer. 2007 Jan 15;96(1):56-60.

9. Concato J, Wells CK. Prostate-specific antigen 'velocity' as a diagnostic test for prostate cancer. J Investig Med. 2006 Nov;54 (7):361-364.

10. Gregorio EP, Grando JP, Saqueti EE, Almeida SH, Moreira HA, Rodrigues MA. Comparison between PSA density, free PSA percentage and PSA density in the transition zone in the detection of prostate cancer in patients with serum PSA between 4 and $10 \mathrm{ng} / \mathrm{mL}$. Int Braz J Urol. 2007 MarApr;33(2): 151-160.

11. Roddam AW, Duffy MJ, Hamdy FC, Ward AM, Patnick J, Price CP, et al. Use of prostate-specific antigen (PSA) isoforms for the detection of prostate cancer in men with a PSA level of 2-10 ng/ml: systematic review and metaanalysis. Eur Urol. 2005 Sep;48(3):386-399; discussion 98-99.

12. Sabbagh R, McCormack M, Peloquin F, Faucher R, Perreault JP, Perrotte P, et al. A prospective randomized trial of 1-day versus 3-day antibiotic prophylaxis for transrectal ultrasound guided prostate biopsy. The Canadian journal of urology. 2004 Apr;11(2):2216-2219.

13. Heijmink SW, van Moerkerk H, Kiemeney LA, Witjes JA, Frauscher F, Barentsz JO. A comparison of the diagnostic performance of systematic versus ultrasound-guided biopsies of prostate cancer. European radiology. 2006 Apr; 16(4):927-938.

14. Cevik I, Dillioglugil O, Zisman A, Akdas A. Combined "periprostatic and periapical" local anesthesia is not superior to "periprostatic" anesthesia alone in reducing pain during Tru-Cut prostate biopsy. Urology. 2006 Dec;68(6):1215-9. 
15. Zackrisson B, Aus G, BeDRdahl S, Lilja H, Lodding P, Pihl CG, et al. The risk of finding focal cancer (less than $3 \mathrm{~mm}$ ) remains high on re-biopsy of patients with persistently increased prostate specific antigen but the clinical significance is questionable. J Urol. 2004 Apr;171(4):1500-1503.

16. Mancuso PA, Chabert C, Chin P, Kovac P, Skyring T, Watt WH, et al. Prostate cancer detection in men with an initial diagnosis of atypical small acinar proliferation. BJU Int. 2007 Jan;99(1): 49-52.

17. Rabets JC, Jones JS, Patel A, Zippe CD. Prostate cancer detection with office based saturation biopsy in a repeat biopsy population. J Urol. 2004 Jul;172(1):94-97.

18. Remzi M, Dobrovits M, Reissigl A, Ravery V, Waldert M, Wiunig C, et al. Can Power Doppler enhanced transrectal ultrasound guided biopsy improve prostate cancer detection on first and repeat prostate biopsy? Eur Urol. 2004 Oct;46(4):451-456.

19. Karaman CZ, Unsal A, Akdilli A, Taskin F, Erol H. The value of contrast enhanced power Doppler ultrasonography in differentiating hypoehoic lesions in the peripheral zone of prostate. European journal of radiology. 2005 Apr;54(1):148-155.

20. Prando A, Kurhanewicz J, BoDRes AP, Oliveira EM, Jr., Figueiredo E. Prostatic biopsy directed with endorectal MR spectroscopic imaging findings in patients with elevated prostate specific antigen levels and prior negative biopsy findings: early experience. Radiology. 2005 Sep;236(3):903-910.

21. Lorente JA, Valenzuela H, Morote J, Gelabert A. Serum bone alkaline phosphatase levels enhance the clinical utility of prostate specific antigen in the staging of newly diagnosed prostate cancer patients. Eur J Nucl Med. 1999 Jun;26(6):625-632.

22. Kosuda S, Yoshimura I, Aizawa T, Koizumi K, Akakura K, Kuyama J, et al. Can initial prostate specific antigen determinations eliminate the need for bone scans in patients with newly diagnosed prostate carcinoma? A multicenter retrospective study in Japan. Cancer. 2002 Feb 15;94(4):964-972.

23. Wymenga LF, Boomsma JH, Groenier K, Piers DA, Mensink HJ. Routine bone scans in patients with prostate cancer related to serum prostate-specific antigen and alkaline phosphatase. BJU Int. 2001 Aug;88(3):226-230.

24. Coakley FV, Qayyum A, Kurhanewicz J. Magnetic resonance imaging and spectroscopic imaging of prostate cancer. J Urol. 2003 Dec;170(6 Pt 2):S6975; discussion S-6.

25. Tempany CM, Zhou X, Zerhouni EA, Rifkin MD, Guint LE, Piccoli CW, et al. Staging of prostate cancer: results of Radiology Diagnostic Oncology Group project comparison of three MR imaging techniques. Radiology. 1994 Jul; 192(1):47-54.

26. Harisinghani MG, Barentsz J, Hahn PF, Deserno WM, Tabatabaei S, van de Kaa $\mathrm{CH}$, et al. Noninvasive detection of clinically occult lymph-node metastases in prostate cancer. N Engl J Med. 2003 Jun 19;348(25):2491-2499.

27. Liu IJ, Zafar MB, Lai YH, Segall GM, Terris MK. Fluorodeoxyglucose positron emission tomography studies in diagnosis and staging of clinically oDRanconfined prostate cancer. Urology. 2001 Jan;57(1):108-111.

28. Klotz L. Active surveillance with selective delayed intervention for favorable risk prostate cancer. Urol Oncol. 2006 Jan-Feb;24(1):46-50.

29. D'Amico AV, Chen MH, Roehl KA, Catalona WJ. Preoperative PSA velocity and the risk of death from prostate cancer after radical prostatectomy. N Engl J Med. 2004 Jul 8;351(2):125-135.

30. Choo R, DeBoer G, Klotz L, Danjoux C, Morton GC, Rakovitch E, et al. PSA doubling time of prostate carcinoma managed with watchful observation alone. Int J Radiat Oncol Biol Phys. 2001 Jul 1;50(3):615-620.

31. Altwein JE. Enhancing the efficacy of radical prostatectomy in locally advanced prostate cancer. Urol Int. 1998;60 Suppl 2:2-10; discussion 35.

32. Hill JR, Fine SW, Zhang J, Eastham JA. Radical prostatectomy for clinical T3 disease: expanding indications while optimizing cancer control and quality of life. Nat Clin Pract Urol. 2007 Aug;4(8):451-454.

33. Salonia A, Suardi N, Crescenti A, Zanni G, Fantini GV, Gallina A, et al. Pfannenstiel versus vertical laparotomy in patients undeDRoing radical retropubic prostatectomy with spinal anesthesia: results of a prospective, randomized trial. Eur Urol. 2005 Feb;47(2):202-208.

34. Malmstrom PU. Lymph node staging in prostatic carcinoma revisited. Acta oncologica (Stockholm, Sweden). 2005;44(6): 593-598.

35. Zietman AL, DeSilvio ML, Slater JD, Rossi CJ, Jr., Miller DW, Adams JA, et al. Comparison of conventional-dose vs high-dose conformal radiation therapy in clinically localized adenocarcinoma of the prostate: a randomized controlled trial. Jama. 2005 Sep 14;294(10):1233-1239.

36. Grills IS, Martinez AA, Hollander M, Huang R, Goldman K, Chen PY, et al. High dose rate brachytherapy as prostate cancer monotherapy reduces toxicity compared to low dose rate palladium seeds. J Urol. 2004 Mar;171(3):1098-1104.
37. Galalae RM, Martinez A, Mate T, Mitchell C, Edmundson G, NuernbeDR N, et al. Long-term outcome by risk factors using conformal high-dose-rate brachytherapy (HDR-BT) boost with or without neoadjuvant androgen suppression for localized prostate cancer. Int J Radiat Oncol Biol Phys. 2004 Mar 15;58 (4): 1048-1055.

38. Bolla M, van Poppel H, Collette L, van Cangh P, Vekemans K, Da Pozzo L, et al. Postoperative radiotherapy after radical prostatectomy: a randomised controlled trial (EORTC trial 22911). Lancet. 2005 Aug 13-19;366(9485): $572-578$.

39. Kumar S, Shelley M, Harrison C, Coles B, Wilt TJ, Mason MD. Neo-adjuvant and adjuvant hormone therapy for localised and locally advanced prostate cancer. Cochrane Database Syst Rev. 2006(4):CD006019.

40. Denham JW, Steigler A, Lamb DS, Joseph D, Mameghan H, Turner S, et al. Short-term androgen deprivation and radiotherapy for locally advanced prostate cancer: results from the Trans-Tasman Radiation Oncology Group 96.01 randomised controlled trial. Lancet Oncol. 2005 Nov;6(11):841-850.

41. Bolla M, van Tienhoven G, de Reijke TM, van den BeDRh AC, van der Meijden AP, Poortmans PM, et al., editors. Concomitant and adjuvant androgen deprivation (ADT) with external beam irradiation (RT) for locally advanced prostate cancer: 6 months versus 3 years ADT-Results of the randomized EORTC Phase III trial 22961. 2007 ASCO Annual Meeting; 2007; Chicago - USA.

42. Cookson MS, Aus G, Burnett AL, Canby-Hagino ED, D’Amico AV, Dmochowski $\mathrm{RR}$, et al. Variation in the definition of biochemical recurrence in patients treated for localized prostate cancer: the American Urological Association Prostate Guidelines for Localized Prostate Cancer Update Panel report and recommendations for a standard in the reporting of suDRical outcomes. J Urol. 2007 Feb;177(2):540-545.

43. Sandler HM, EisenbeDRer MA. Assessing and treating patients with increasing prostate specific antigen following radical prostatectomy. J Urol. 2007 Sep;178(3 Pt 2):S20-24.

44. Maximum androgen blockade in advanced prostate cancer: an overview of the randomised trials. Prostate Cancer Trialists' Collaborative Group. Lancet. 2000 Apr 29;355(9214):1491-1498.

45. Seidenfeld J, Samson DJ, Hasselblad V, Aronson N, Albertsen PC, Bennett $\mathrm{CL}$, et al. Single-therapy androgen suppression in men with advanced prostate cancer: a systematic review and meta-analysis. Annals of internal medicine. 2000 Apr 4;132(7): 566-577.

46. Miller K, Steiner U, Lingnau A, Keilholz U, Witzsch U, Haider A, et al., editors. Randomised prospective study of intermittent versus continuous androgen suppression in advanced prostate cancer. 2007 ASCO Annual Meeting; 2007; Chicago - USA.

47. Ryan CJ, Small EJ. Role of secondary hormonal therapy in the management of recurrent prostate cancer. Urology. 2003 Dec 29;62 Suppl 1:87-94.

48. Tannock IF, de Wit R, Berry WR, Horti J, Pluzanska A, Chi KN, et al Docetaxel plus prednisone or mitoxantrone plus prednisone for advanced prostate cancer. N Engl J Med. 2004 Oct 7;351(15):1502-1512.

49. Kaasa S, Brenne E, Lund JA, Fayers P, Falkmer U, HolmbeDR M, et al. Prospective randomised multicenter trial on single fraction radiotherapy $(8 \mathrm{~Gy}$ $\mathrm{x}$ 1) versus multiple fractions (3 Gy x 10) in the treatment of painful bone metastases. Radiother Oncol. 2006 Jun;79(3):278-284.

50. Saad F, Gleason DM, Murray R, Tchekmedyian S, Venner P, Lacombe L, et al. Long-term efficacy of zoledronic acid for the prevention of skeletal complications in patients with metastatic hormone-refractory prostate cancer. Journal of the National Cancer Institute. 2004 Jun 2;96(11):879-882.

51. Sartor O, Reid RH, Hoskin PJ, Quick DP, Ell PJ, Coleman RE, et al. Samarium-153-Lexidronam complex for treatment of painful bone metastases in hormone-refractory prostate cancer. Urology. 2004 May;63(5):940945.

52. Morote J, Morin JP, Orsola A, Abascal JM, Salvador C, Trilla E, et al. Prevalence of osteoporosis during long-term androgen deprivation therapy in patients with prostate cancer. Urology. 2007 Mar;69(3):500-504.

Correspondencia autor: Dr. Leonardo Oliveira Reis

Sección de Urología Oncológica

Discipline of Urology, Universidad de Campinas

E-mail autor: reisleo@unicamp.br

Información artículo: Revisión - Cáncer de próstata

Trabajo recibido: diciembre 2008

Trabajo aceptado: enero 2009 